\title{
Successful in vitro Fertilization Using Autologous Gametes after Eight Failed Trials
}

\author{
Ahmed M. Isa ${ }^{1 *}$ \\ ${ }^{I}$ Assisted Conception Unit, Obstetrics and Gynecology Department, College of Medicine, King Saud University, \\ Riyadh, Saudi Arabia.
}

\begin{abstract}
:
Background: Many infertile couples worldwide resort to donated gametes to overcome their infertility problems, however many others cannot for cultural or religious reasons. They have no other options but probably to keep trying In-Vitro Fertilization (IVF) treatment using their own gametes. We wanted to see if we would be able at some point to help such cases fulfil their dreams.

Case Report: Four infertility cases with a history of many unsuccessful IVF trials; eight, seven, six, and four, suggestively because of very poor fertilization and/or poor embryo development due to sever male factor that ranged from azoospermia to severe oligo-terato-asthenozoospermia. Yet, in their subsequent trials we managed to help them conceive with live births at the end.

Conclusion: In some complex infertility cases, when the use of donor-gametes is not an option, probably persistent hopefulness in IVF treatment with autologous gametes stays as the solution.

Keywords: Infertility, Male Factor, Gametes, Insemination, In-Vitro Fertilization, Pregnancy Rate.

\section{Introduction}

In Vitro Fertilization (IVF) has been prescribed as a sub-fertility treatment since the first successful test tube baby achieved by Steptoe and Edwards in 1978 (1). The improvements in the assisted reproductive technology (ART), in general, has been ongoing since then to cope with the complicated prognoses of a good percentage of the sub-fertile patients. Yet, many of these cases, and after undergoing a number of unsuccessful IVF cycles, seem to have to resort to donated gametes as the only currently available solution for their infertility. There is still a long way to go until artificial germ cells (from stem cells) will be used in the clinical practice (2). However, a good percentage of infertile couples worldwide just cannot use donated gametes because of variety of reasons due to traditional, ethical, emotional, or religious reasons. For those, and until the current moment, the only available option for them is probably to keep trying IVF treatment with their own gametes, and from this category we have the following successful cases to report.
\end{abstract}

\section{Cases Reports}

Case 1: A 27-year-old woman was referred to our clinic with 11 years of secondary infertility. Her husband suffered from a severe oligo-terato-asthenozoospermia, while she suffered only from a blockage in her left Fallopian tube. She had a history of a total of eight IVF failed trials in other different clinics, one of which ended with ectopic pregnancy that was removed through a salpingectomy operation. All eight previous IVF cycles resulted in poor fertilization and/or poor embryo development. The patient had a regular menstrual cycle every 28 days, and had also a normal hormone profile; follicular stimulating hormone (FSH) was $6.2 \mathrm{IU} / \mathrm{L}$, luteinizing hormone $(\mathrm{LH})$ was $4.88 \mathrm{IU} / \mathrm{L}$, prolactin was $355 \mathrm{mIU} / \mathrm{L}$, while her thyroid stimulating hormone (TSH) was $3.35 \mathrm{mIU} / \mathrm{L}$.

In her ninth IVF treatment cycle a short ovarian stimulation (OS) protocol was employed. Starting from the second-day of her period, the patient received two daily subcutaneous injections; one was a 150 IU of the human menopausal gonadotropin (HMG) Menogon, and the second was a $0.1 \mathrm{mg}$ of Decapeptyl, a gonadotropin releasing hormone $(\mathrm{GnRH})$ agonist, both were produced by Ferring Pharmaceuticals, Inc., USA. The growth of the follicles was monitored by ultrasound examinations. When four of her nine growing follicles reached a mean diameter of $18 \mathrm{~mm}$, the patient was instructed to take a one dose of intramuscular injection (IM) of the human chorionic gonadotropin (hCG), 10,000 IU (ACON Pharmaceuticals, Cyprus, Europe) 36 hours before the eggs retrieval (ER) (3) which was done using a 17-gauge single lumen needle (Cook, Queensland, Australia).

Eight cumulus oocyte complexes (COCs) were received and incubated in culture medium for two hours at $37^{\circ} \mathrm{C}$, and $5 \% \mathrm{CO}_{2}$ before they were denuded in preparation for the intracytoplasmic sperm injection (ICSI) one hour later. All eight oocytes were normally fertilized and kept in culture till day four where three compacted, grade 1 , embryos were easily transferred back to the patient's uterus. Fourteen days later, the patient's blood serum hCG was $110 \mathrm{IU} / \mathrm{L}$ announcing a successful pregnancy. Six weeks later the ultrasound examination showed two 
healthy viable gestational sacs. Two healthy babies, a boy and a girl were born through a cesarean section after thirty-six weeks of pregnancy, with no complications, and they are doing great since then.

Case 2: A 36-year-old woman who suffered from polycystic ovarian syndrome (PCOS) and whose husband suffered from severe oligo-terato-asthenozoospermia, too. The couple had seven years of secondary infertility, during which they underwent seven unsuccessful IVF trials, the last two of which were in our clinic. They usually produced poorly developed embryos of grade- 2 or 3 in all failed cycles.

In her eighth IVF cycle, the third with us, she produced 21 oocytes, 16 of which were MII that were all fertilized by ICSI. Luckily this time three embryos made it to grade-1 blastocyst stage and were all transferred, because of the history of many failed trials and because of the patient's age, while all other embryos were poorly developed and were arrested between the 2-cell and 12-cell stages. The patient has an ongoing pregnancy of 16-weeks thus far.

Case 3: A 28-year-old woman whose husband suffered from azoospermia was referred to our clinic with seven years of primary infertility during which they have had undergone six unsuccessful IVF cycles in other IVF clinics. Examination of the woman along with her hormonal profile showed that she was not suffering from any cause of infertility. Testicular biopsy extraction (TESE) was done and very few sperms were detected and frozen.

Upon ER eight oocytes were collected, seven of which were mature and ICSI was done to them with seven twitching sperms. Five eggs were normally fertilized and cultured where two of which reached the blastocyst stage (grade-1), while a third embryo was a grade-2 blastocyst. All three blastocysts were transferred because of the previous repeated failure. This cycle resulted in a successful and completely normal pregnancy that reached full term and now their son is two years old and the couple is back for another IVF cycle.

Case 4: A 29-year-old female who suffered from PCOS and whose husband suffered from severe oligo-teratoasthenozoospermia. They had primary infertility for five years during which they underwent four IVF cycles in our clinic. All four cycles resulted in a number of eggs ranged between 8 to 18 mostly mature oocytes. ICSI was performed and resulted in very poor normal fertilization, 2-3 oocytes, and poor embryo development as well.

In their fifth cycle in our clinic as well, 9 eggs were retrieved, 6 of which were mature where ICSI was done to them. Two eggs only were normally fertilized, but luckily they developed into two compacted (day-4), grade-1, embryos that were transferred, and that resulted in a successful normal pregnancy, that was delivered normally at a full term of 40 weeks. Their completely normal girl is already two years old.

\section{Discussion}

In Vitro Fertilization (IVF) has become the infertility miraculous solution for many couples who otherwise would have been considered as absolutely infertile. Clinical and scientific researches have been ongoing to optimize the fertilization and the embryo culturing conditions that emulate the natural in-vivo conditions for better pregnancy rates. Yet, in many cases and after trying the IVF for a few times the couples find themselves forced to resort to using donor gametes, either eggs or sperms, instead of theirs, for better chances of IVF outcome (4). Although using donor gametes have become an acceptable solution to many couples around the world, yet a good percentage of the world population cannot use donated gametes for cultural, religious, or even just personal reasons. Hence they have no other options but probably to keep trying IVF treatment with their own gametes hoping for the miraculous success. The question however remains; when to keep trying and when to stop especially with the IVF treatment increasingly high cost? Luckily our clinic, as well as a few other clinics, is fully supported by the government of Saudi Arabia, which gives such cases no worry about the treatment cost.

All four cases mentioned above were common in that the couples experienced repetitive failed IVF trials either in our clinic or in IVF clinics elsewhere, some of which were even abroad. They have failed eight times, seven times, six times, and four times, respectively. They all suffered from severe male factor infertility that ranged from azoospermia to severe oligo-terato-asthenozoospermia with no option of using donor sperm because of cultural and religious reasons, as it is known in all countries of the Middle East and probably in other regions of the world. As clinicians, we also had to offer them, like the rest of our patients, full moral support and the best clinical service we possibly could, suppressing any feels of hopelessness and thinking of any probable tips that might enhance the final results and help them conceive and fulfill their dreams. Thankfully we succeeded to reach the happy end, sometimes from the first trial in our clinic, or after we tried and failed ourselves before we achieve the long-awaited success of full term gestation and the delivery of normal children in three of the above mentioned cases, while the fourth has still an ongoing normal pregnancy of sixteen weeks. Janssens, RMJ et al. (1999) (5) reported a successful natural IVF cycle that ended with a healthy live birth after four failed attempts with stimulated cycles plus two failed natural cycles as well. 


\section{Conclusion}

When the use of donor gametes is not an option, more IVF trials with autologous gametes could still result in successful pregnancy in spite of having failed many times before.

\section{Acknowledgements}

The author would like to appreciate the College of Medicine Research Center, the Deanship of Scientific Research, King Saud University, for supporting this work. The author would like also to thank all the IVF team of the Assisted Conception Unit at King Khalid University Hospital in Saudi Arabia for their dedication and professionalism in serving our patients.

\section{Conflict of Interest:}

The authors would like to announce no conflict of interest with any institution or anybody.

\section{References}

[1]. P.C. Steptoe, and R.G. Edwards, Birth after the preimplantation of a human embryo. Lancet, ii, $1978,336$.

[2]. I. Moreno, J.M. Miguez-Forjan, and C. Simon, Artificial gametes from stem cells. Clin Exp Reprod Med. 2015, Jun; 42(2): 33-44.

[3]. L. De Lauretis, C. Scarduelli, U. Bailo, et al., IVF in natural cycle: our experience. Hum. Reprod. 9 (Suppl. 4) 1994, 131.

[4]. J.F. Kawwass, M. Mansour, S. Crawford, D.M. Kissin, D.R. Session, A.D. Kulkami, D.J. Jamieson, Trends and outcomes for donor cycles in the United States, 2000-2010. JAMA. 2013, Dec. 11; 310(22): 2426-2434.

[5]. R.M.J. Janssens, C.B. Lambalk, R. Schats, J. Schoemaker, Successful in-vitro fertilization in a natural cycle after four previously failed attempts in stimulated cycles. Hum. Reprod. 1999, 14(10): 2497-2498. 